Bedrii Nataliia. Adaptive griffiths scale - modern experience in assessing the psychomotor development of preschool children. $\begin{array}{llllll}\text { Journal of Education, Health and Sport. 2021;11(11):276-285. } & \text { eISSN }\end{array}$ http://dx.doi.org/10.12775/JEHS.2021.11.11.027

https://apcz.umk.pl/JEHS/article/view/JEHS.2021.11.11.027

https://zenodo.org/record/5796293

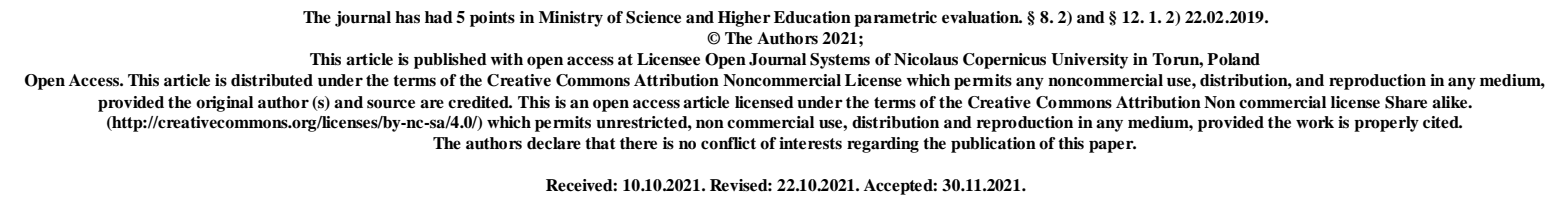

Recived: 10102021. Revised: 22.102021. Accepted: 30.11.2021.

UDC: 616-053.4:159.943

\title{
ADAPTIVE GRIFFITHS SCALE - MODERN EXPERIENCE IN ASSESSING THE PSYCHOMOTOR DEVELOPMENT OF PRESCHOOL CHILDREN
}

\author{
Nataliia Bedrii
}

\author{
National Pirogov Memorial Medical University, Vinnytsya \\ Pediatrics department Nr. 1, Vinnytsya, Ukraine
}

Abstract

Premature newborns build a specific category of children due to their innate morphofunctional immaturity and specific pathological conditions that determine significant survival, morbidity and postnatal care results as compared to children of other weight categories. In the long-term adverse effects of preterm birth, the incidence of neuropathy remains high and requires further improvements in diagnostic methods and timely corrections for a better developmental prognosis. Although over the past three decades, numerous tools have been invented to predict long-term adverse effects of preterm neonates as well as methods for determining degree of disorders, neither these tools nor their weak and strong points have undergone a comprehensive overview. Aim of the research was to improve long-term preterm birth outcomes by using the adaptive Griffiths scale for preschoolers. The psychomotor development of 105 preschoolers was evaluated. Assessment of indicators of psychomotor development was carried out by the adaptive Griffiths scale and the conventional method. In the course of the study, this category of children was under doctors' from Neonates Postdischarge Follow-ups Department with systematic evaluation of their health condition. 
Dynamic examination was performed during the first year of life once per 3 months, from the 2nd to the 6th year of life - corresponding to an individual rehabilitation plan. Patients $(n=105)$ were divided into 3 groups depending on the severity of the disabling pathology.

The analysis of indicators of psychomotor development (by traditional methods) of prematurely born preschoolers, divided into 3 groups in accordance with severity degree of disabling pathology:

Group A children with severe long-term consequences that led to disability - 54 $(51.4 \%)$

Group B. Children with long - term consequences who are subject to correction - 24 $(22.8 \%)$

Group C. Children with long-term consequences not significantly affecting their health condition $-27(25.7 \%)$.

The conducted survey suggests, that above $70 \%$ of the children had various patterns of delayed psychomotor development. The assessment of psychomotor development showed that the cause structure of delayed psychomotor development of preschoolers has the same trend according to different examination methods, but the assessment by the adaptive Griffiths scale shows more accurate values for each patient, enables quick detection of long-term adverse consequences of premature birth, recommendations for parents for neonates' rehabilitation and habilitation, as well as examination in dynamics.

In the flow of the research, the adaptive Griffiths scale of psychomotor development proved to be a valid diagnostic tool for determining indicators of preschoolers' psychomotor development, estimating their intellectual coefficient and forming individual patient's profile.

Keywords: premature newborns; psychomotor development preschoolers; Neonates Post-discharge Follow-ups Department; disability; long-term consequences of premature birth

Introduction. Premature newborns are a group of increased risk of severe pathology development [1, 4]. World studies show that long-term consequences and deterioration of life quality of prematurely born children are determined by chronic pathology, developing as a result of diseases transferred in the first months of life, intensive care and iatrogenic exposure, as well as antenatal development, in particular, multi-vector inflammatory changes and pathological immaturity of the placenta $[2,11,15]$. 
In the long-term adverse effects of preterm birth, the incidence of neuropathy remains high and requires further improvements in diagnostic methods and timely corrections for a better future developmental prognosis $[9,12,15]$.

Psychomotor development of a child is a fairly broad concept, which includes the following areas:

- $\quad$ development of motor functions (large motor skills);

- development of hand dexterity, coordination of movements (fine motor skills);

- development of active speech and its understanding;

- comprehending relationship between phenomena and objects (development of thinking);

- ability to communicate with people (development of social and communicative functions);

- developing independence of actions.

Child's development is a continuous process that passes three obligatory stages [13]. Child's development is defined by the concepts of speed or pace and maturation. The pace characterizes the intensity of the processes of age-related development of the nervous and other systems of the body at a certain stage. The pace of age-related transformations fluctuates during development, and the child experiences periods of acceleration and deceleration of biological maturation.

Assessment of a child's neuropsychiatric development includes both neurological examination and determination of the ability to interact with the environment, i.e. identification of behavioral reactions. There are many domestic and foreign methods for assessing psychomotor development, based on the mandatory identification of various developmental patterns, which contributes to the timely identification of the causes of lag and further correction of identified disorders. Despite numerous tools developed over the past three decades to predict long-term adverse outcomes of preterm neonates and as well as methods for determining degree of disorders, no study has provided a comprehensive overview of these tools, as well as their strong and weak points [6, 7]. These include: Peabody Development Motor Scale-2, Bailey scales of Infant Development (BSID III), Denver development screening test (DDST), Wechsler Preschool and Primary scale of Intelligence (WPPSI), Leiter-3 (International Leiter performance scale).

All of the above methods are standardized and valid, but rather costly, have a limited age range and narrow focus which hinders comprehensive assessment of indicators of psychomotor development. 
A well-known standardized method for assessing psychomotor development is the adaptive Griffiths scale [8]. This method was first published in 1954 by Dr. Ruth Griffiths and has had several revisions. The method is standardized, evaluates both the motor and cognitive spheres; due to the wide age range allows to provide evaluation in dynamics.

Important is the method of early intervention aimed at providing psychological and pedagogical assistance to children at early stages of development, providing assistance to families of high-risk category neonates; advisory activities; selecting adequate ways to interact with the child; correcting developmental disorders; rehabilitation and habilitation [10, 14].

Aim of the research. To improve long-term preterm birth outcomes by using the adaptive Griffiths scale for preschoolers.

Materials and methods of research. The psychomotor development of 105 preschoolers prematurely born with a body weight of less than $1500 \mathrm{~g}$ was evaluated. Assessment of indicators of psychomotor development was carried out by the adaptive Griffiths scale (copyright Certificate No. 107640 "scale for evaluating indicators of psychomotor development in preschool children") and the standard method (using the following tools: graphic explanations, schematic images, tabular chart tasks for preschoolers) [16].

The adaptive Griffiths scale is designed for children aged 2 years to 6 years and 11 months. It comprises 5 subscales: motor skills, social adaptation, hearing and speech, visual function, hand motor function (fine motor skills), as well as the ability to learn. Each pattern is evaluated in scores. After completing the tasks, the child's intelligence coefficient is calculated and the child's development is determined in correspondence to the age. Thanks to the wide age range, it is possible to conduct dynamic monitoring of the patient and, if necessary, evaluation in dynamics. It is worth noting that the child may be ahead of the standard indicator by a certain criterion. In this case, a score for the succeeding age is chosen. Conversely, in case of developmental delay a score for preceding age is chosen.

In the course of the study, this category of children was under doctors' catamnestic observation with systematic evaluation of their health condition. Clinical indicators such as body weight, height, head circumference, neurological and intellectual development, the presence of somatic pathology, including diseases of the respiratory system, musculoskeletal system and hematological problems, were analyzed. Ophthalmological and audiological examinations were performed dynamically to assess vision and hearing [5]. 
Patients $(n=105)$ were divided into 3 groups depending on the severity of the disabling pathology: group 1 - children with severe disabling consequences $(n=54)-51.4 \%$, group $2-$ children with adverse consequences who are subject to correction $(n=24)-22.8 \%$, group 3 included healthy children without signs of disabling pathology $(n=27)-25.7 \%$.

The analysis of indicators of psychomotor development (by conventional methods) of prematurely born preschoolers was conducted. These were divided into 3 groups in accordance with severity degree of disabling pathology (see the data in Diagram 1):

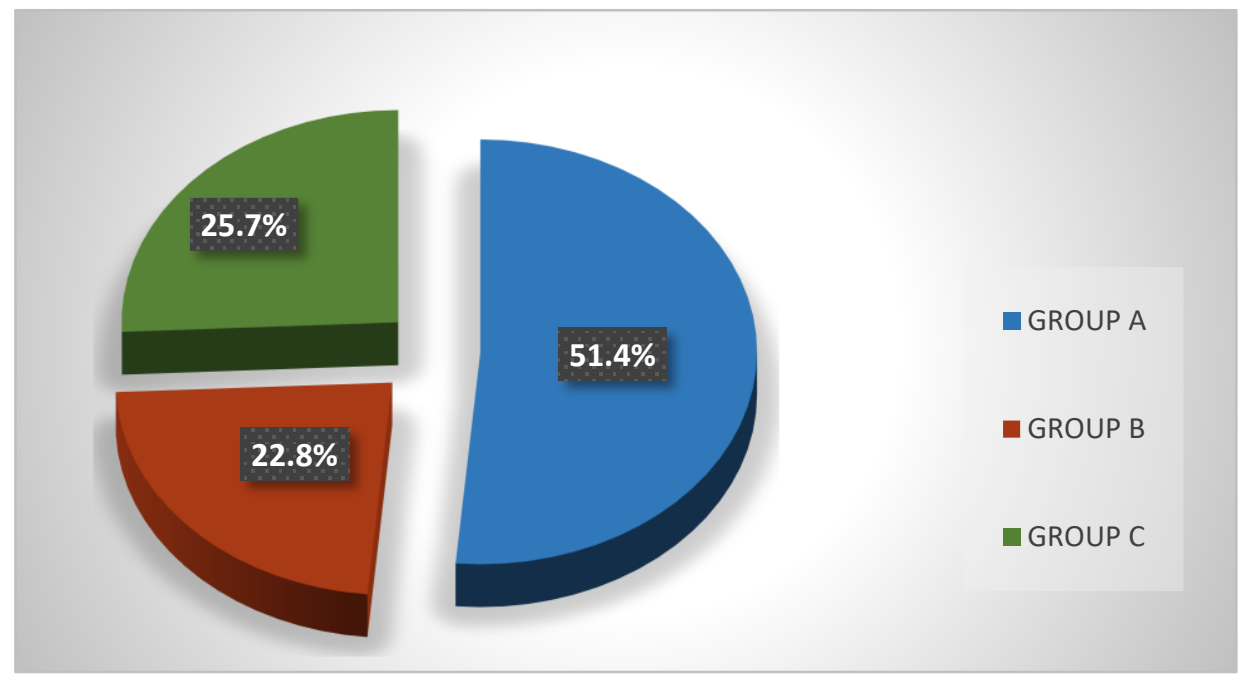

Diagarama 1. Analysis of indicators of psychomotor development of prematurely born preschoolers, conducted by the traditional method

Post-discharge Follow-ups examination was performed during the first year of life once per 3 months, from the 2 nd to the 6 th year of life - corresponding to an individual rehabilitation plan [3].

Diagnoses were established on the basis of clinical, laboratory, instrumental (ultrasound, radiological) and bacteriological studies in accordance with ICD-10. The work was carried out on the basis of the Municipal non-commercial enterprise of the Vinnytsia regional children's Clinical Hospital of the Vinnytsia regional council, city Vinnytsia, Ukraine.

In the course of the work, the following research methods were used: clinical and anamnestic, instrumental: neurosonography (NSG), magnetic resonance imaging (MRI), electroencephalography (EEG), hearing screening by optoacoustic emission and audiometry, determination of visual acuity by a binocular ophthalmoscope with special lenses, the method of visual evoked potentials in the newborn period and using special charts in subsequent age periods), anthropometric methods. To solve the tasks set and check the initial assumptions, 
the obtained results were processed by a number of statistical methods of the Microsoft Excel 2010 (14.0.6024.1000) SP 1 MSO (14.0.6023.1000) software system.

\section{Conclusion of the Bioethics Commission}

The work was carried out in accordance with the principles of the Helsinki Declaration and the recommendations of the International Council for the harmonization of good clinical practice. The consent of the patient's parents was obtained for the study. The study was approved by the committee on Bioethics at the National Pyrogov Memorial Medical University, Vinnytsya.

\section{Research results and their discussion}

The children under study were examined by the Griffith method to determine indicators of psychomotor development, calculate their intelligence coefficient, and determine the cause of the delayed psychomotor development. (Figure 2).

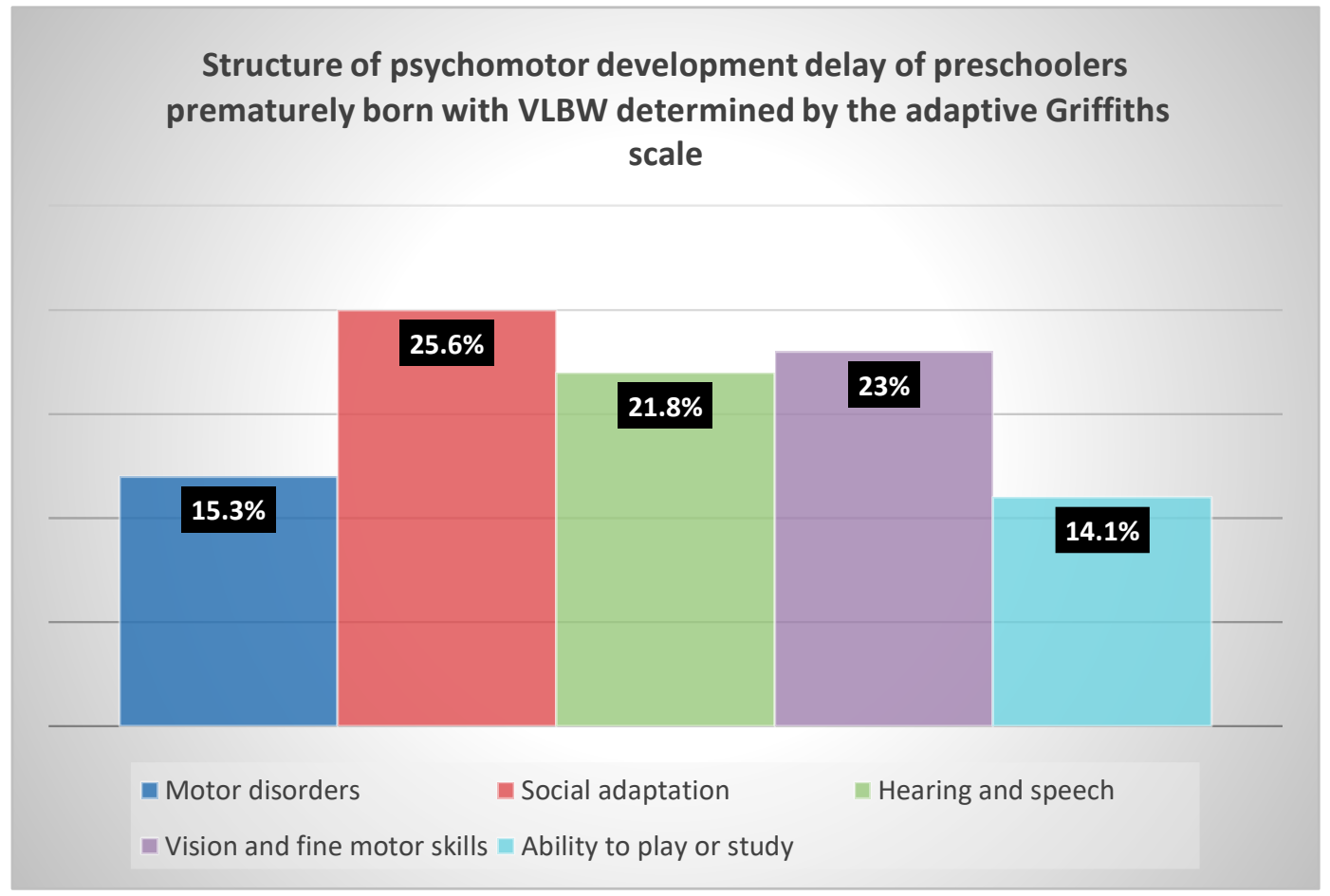

Figure 2. The structure of the causes of delayed psychomotor development in prematurely born preschoolers with VLBW (very low body weight) was determined by the adaptive Griffiths scale

The results showed that more than $70 \%$ of the children under study had equivalents of delayed psychomotor development of various patterns. Thus, the analysis of the causes of delayed psychomotor development of preschoolers showed that disorders of social adaptation rank top - $20(25.6 \%)$ children, almost the same frequency had visual and auditory disorders - 
$18(23 \%)$ and $17(21.8 \%)$ children, respectively, while motor disorders - $12(15.3 \%)$ and the ability to play and/or learn $11(14.1 \%)$ are the least common among the problems of this group category.

In parallel, the assessment of psychomotor development of this group was carried out by a conventional method (diagram 3 ).

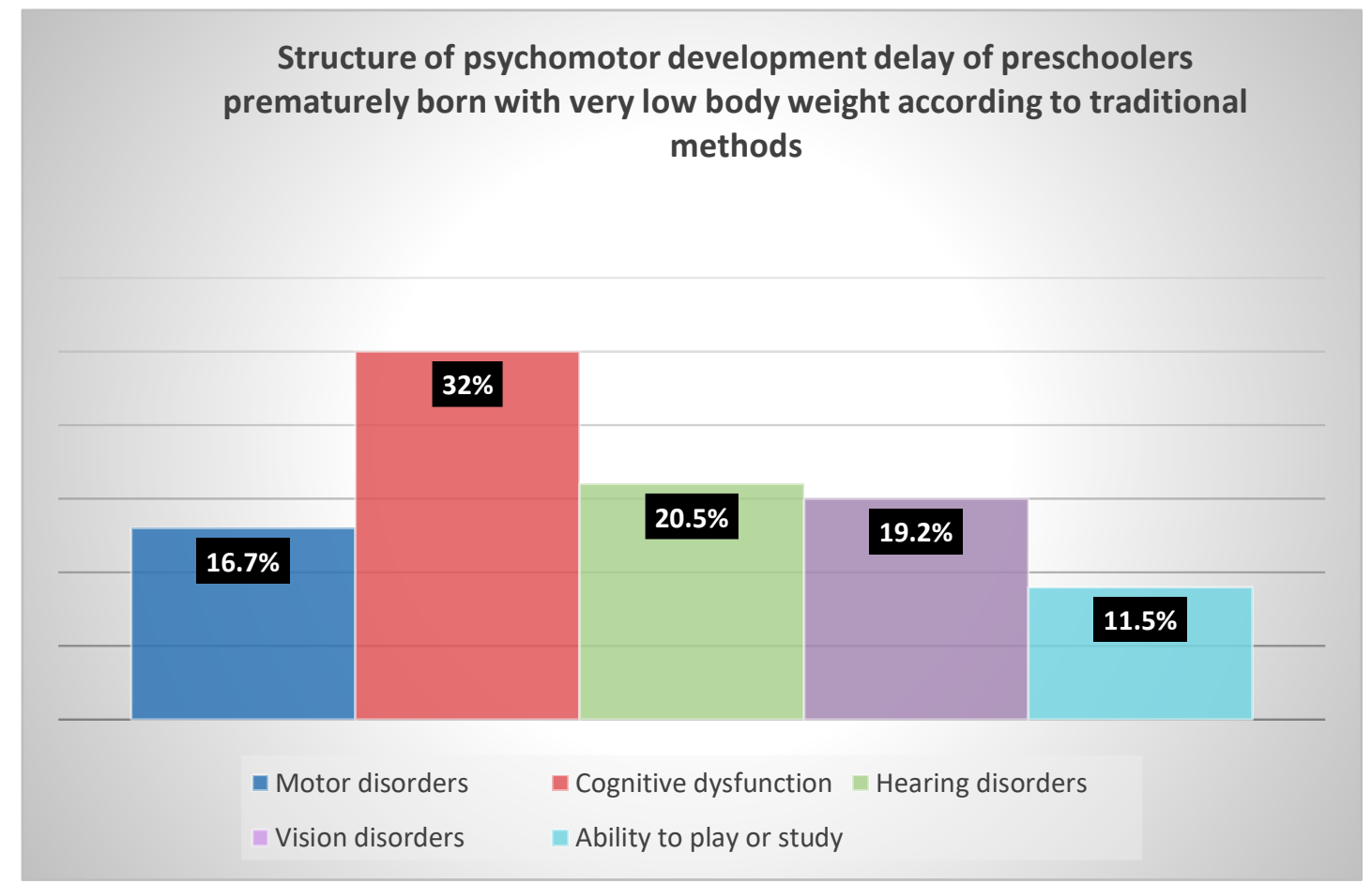

Figure 3. The structure of the causes of delayed psychomotor development in prematurely born preschoolers with VLBW (very low body weight) determined by the traditional methods

Analysis of the causes of psychomotor development disorders among prematurely born preschoolers with VLBW shows that out of 105 patients examined, 25 (32\%) had cognitive dysfunctions, $16(20.5 \%)$ and $15(19.2 \%)$ had audiological and ophthalmological disorders, respectively, motor disorders were observed in $13(16.7 \%)$ patients, and the discrepancy in the ability to play corresponding to the age was identified in 9 (11.5\%) children.

The analysis of the causes of delayed psychomotor development of preschoolers found no significant differences between both methods of evaluation ( $\mathrm{p}>0.05)$. Thus, $12(15.3 \%)$ children examined by the Griffiths scale presented motor disorders compared to $13(16.7 \%)$ examined by the traditional method ( $\mathrm{p}>0,05) ; 11(14.1 \%)$ and $9(11.5 \%)$ examined patients had impaired play and/or learning skills, respectively $(\mathrm{p}>0,05)$; while ophthalmic and 
audiological problems were diagnosed in $18(23 \%)$ and $17(21 \%)$ children using an adaptive method, compared to $15(19.2 \%)$ and $16(20.5 \%)$ examined by the standard method, which indicates the absence of difference $(p>0,05)$. There are no significant differences in the frequency of cognitive component dysfunctions in patients examined by the Griffiths scale $20(25.6 \%)$ and those examined by conventional methods - 25 (32\%) children, respectively. But this indicates that the assessment by the adaptive Griffiths scale gives more accurate results, enables to quickly detect long-term adverse consequences of premature birth, give recommendations for families of such children on forming an individual rehabilitation and habilitation program, and examine them in dynamics.

\section{Conclusions}

1. In the flow of the research, the adaptive Griffiths scale of psychomotor development proved to be a valid diagnostic tool for determining indicators of preschoolers' psychomotor development, estimating their intellectual coefficient and forming individual patient's profile.

2. Assessment of the psychomotor development of prematurely born preschool children is an important component of the early intervention program, formation of an individual rehabilitation program and taking measures for habilitation in society

Prospects for further research consist in studying the health status of children in catamnesis, providing psychological and pedagogical assistance to neonates at the early stages of care; developing advisory activities; selecting adequate ways to interact with the child; correcting developmental disabilities; rehabilitation and habilitation

Conflict of interest: The authors did not state any conflict of interest.

Sources of funding. Own.

\section{References:}

1. Avilov O. V., Kosymov E.A., Vanin E. Yu. \& Rybakova O. V. (2017). Risk factors for developing disability in children born with extremely low and very low body mass. Medical and Pharmaceutical Journal "Pulse", 19(12), 63-67.

2. Arkhipova Maria Yuryevna \& Zakharova Svetlana Yuryevna (2016). Assessment of the health status of extremely pre-term infants. Russian Bulletin of Perinatology and pediatrics, 61 (1), 32-36.

3. Volyanyuk E. V., Safina A. I., Khuzieva G. M., Potapova M. V., \& Sharipova O. V. (2016). Subsequent observation of premature babies in the city center of catamnesis of the city of Kazan. Practical medicine, (8(100)). 
4. Gasyuk, N. I. (2015). Assessment of psychomotor development of prematurely born children in the first year of life. Actual problems of modern medicine: Bulletin of the Ukrainian medical dental Academy, 15(3-1 (51)).

5. Dobryansky D. O., Menshikova A. O. \& Borisyuk O. P. (2019). Long-term consequences of bronchopulmonary dysplasia in premature infants. Modern Pediatrics, 4(100). doi https://doi.org/10.15574/SP.2019.100.43

6. Znamenskaya, T. K., Nikulina, L. I., Rudenko, N. G., \& Vorobyova, O. V. (2017). Analysis of the work of perinatal centers in the care of prematurely born children in Ukraine. Neonatology, surgery and Perinatal Medicine, (7,№ 2), 5-11. https://doi.org/10.24061/2413-4260.VII.2.24.2017.1

7. Znamenskaya, T. G., \& Vorobyova, O. V., \& Dubinina, T. Yu. (2017). Strategic directions of reconstruction of the healthcare system of newborns and children in Ukraine. Neonatology, surgery and Perinatal Medicine, (7,№ 4), 5-12. https://doi.org/10.24061/2413-4260.VII.2.24.2017.1

8. Pokhylko, V. I., Traverse, G. M., Tsvirenko, S. M., Zhuk, L. A., \& Oskomenko, M. M. (2016). Premature babies: a modern view of postnatal adaptation and health at an early age. Bulletin of problems of biology and medicine, 2(1).

9. Riga, Oh. O., Gordienko,I. V., Marabyan, R. V., Khotsenko, G. O., \& Bezrukova, Yu. O. (2015). Health status and features of sending prematurely born children to rehabilitation programs during the first three years of life. Modern Pediatrics, (4), 33-36.

10. Yablon, O.. S., Bondarenko, T. V., Savrun, T. I., Bykovskaya, O. A., Moravskaya, O. A., Gomon, R. O., ... \& Nazarchuk, N. Moscow (2018). Experience of the catamnestic Observation Center in the Vinnytsia region.

11. Cheong, J., Burnett, A. C., Treyvaud, K., \& Spittle, A. J. (2020). Early environment and long-term outcomes of preterm infants. Journal of neural transmission (Vienna, Austria : 1996), 127(1), 1-8. https://doi.org/10.1007/s00702-019-02121-w

12. Griffiths, A., Toovey, R., Morgan, P. E., \& Spittle, A. J. (2018). Psychometric properties of gross motor assessment tools for children: a systematic review. $B M J$ open, 8(10), e021734. https://doi.org/10.1136/bmjopen-2018-021734

13. Griffiths, A., Morgan, P., Anderson, P. J., Doyle, L. W., Lee, K. J., \& Spittle, A. J. (2017). Predictive value of the Movement Assessment Battery for Children - Second Edition at 4 years, for motor impairment at 8 years in children born preterm. Developmental medicine and child neurology, 59(5), 490-496. https://doi.org/10.1111/dmcn.13367 
14. Riga, O., \& Zbrozhik, E. (2014). Preconditions for creating a catamnestic follow-up system for newborns at risk. Neonatology, surgery and Perinatal Medicine, 4(3 (13)), 55-60. https://doi.org/10.24061/2413-4260.IV.3.13.2014.10

15. Yablon, O. S., Savrun, T. I., Nazarchuk, N. M., Serheta, D. P., Kholod, L. P., \& Dzema, I. O. (2020). Features of the course of the neonatal period in prematurely born children with a body weight $<1500 \mathrm{~g}$, depending on the state of the placenta. Neonatology, Surgery And Perinatal Medicine,10(2 (36)), 37-44. https://doi.org/10.24061/2413$\underline{4260 . X .2 .36 .2020 .4}$

16. https://zakon.rada.gov.ua/rada/file/v0149282-08 